\title{
Clinical features and survival analysis of 97 coronavirus disease 2019 (COVID-19) patients
}

\author{
Aixin $\mathrm{Li}^{1}$, Feng Chen ${ }^{2}$, Yue Gao ${ }^{1}$, Xiaojie Huang ${ }^{3}$, Tongzeng Li $^{4}$, Jiaying Zhang ${ }^{4}$, Lianchun Liang ${ }^{4}$, \\ Xuemei $\mathrm{Li}^{5}$ \\ ${ }^{1}$ Center for Infectious Diseases, Beijing Youan Hospital, Capital Medical University, Beijing, China; ${ }^{2}$ Department of Radiology, Beijing Youan \\ Hospital, Capital Medical University, Beijing, China; ${ }^{3}$ Department of Infection and Immunological Medicine, Beijing Youan Hospital, Capital \\ Medical University, Beijing, China; ${ }^{4}$ Department of Respiratory and Infectious Diseases, Beijing Youan Hospital, Capital Medical University, Beijing, \\ China; ${ }^{5}$ Department of Chronic Disease Management Center, Beijing Youan Hospital, Capital Medical University, Beijing, China \\ Contributions: (I) Conception and design: A Li, X Li; (II) Administrative support: L Liang, X Li; (III) Provision of study materials or patients: None; (IV) \\ Collection and assembly of data: A Li, F Chen, Y Gao; (V) Data analysis and interpretation: A Li, X Huang, T Li, J Zhang; (VI) Manuscript writing: \\ All authors; (VII) Final approval of manuscript: All authors. \\ Correspondence to: Lianchun Liang. Department of Respiratory and Infectious Diseases, Beijing Youan Hospital, Capital Medical University, Beijing \\ 100069, China. Email: 1lc671215@sohu.com; Xuemei Li. Department of Chronic Disease Management Center, Beijing Youan Hospital, Capital \\ Medical University, Beijing 100069, China. Email: 1xm801@ccmu.edu.cn.
}

Background We aim to investigate the clinical characteristics and survival rate of coronavirus disease 2019 (COVID-19) patients.

Methods: Ninety-seven COVID-19 patients were enrolled. The laboratory results, lung imaging and medical treatment were compared. Patients were followed up after 1 year, and the Kaplan-Meier test was used for survival analysis.

Results: Compared with the non-severe group, the age of the severe group was older, and the proportion of concomitant diseases were higher. As fever was the primary clinical manifestation, dyspnea and anorexia were more common in severe patients. Lung imaging manifestations and laboratory indicators were worse in the severe group. Accordingly, the treatment of glucocorticoid, antibiotics, and advanced life support were in high proportion. Of the 97 patients with COVID-19, 4 severe patients died within one month during the 1-year follow-up, with the median survival time of 47.0 weeks (95\% CI: 45.1-48.9).

Conclusions: Severe cases of COVID-19 are characterized by advanced age, more concomitant diseases and complications, which lead to a decreased short-term survival rate. However, there were no deaths after one month, which implied a good prognosis if the risk period were passed smoothly.

Keywords: Coronavirus disease 2019 (COVID-19); survival analysis; age; concomitant diseases

Submitted Feb 19, 2021. Accepted for publication Jun 01, 2021.

doi: $10.21037 /$ apm-21-393

View this article at: https://dx.doi.org/10.21037/apm-21-393

\section{Introduction}

Coronavirus disease 2019 (COVID-19) has posed an international threat to public health as the novel coronavirus swept through the globe (1). The pathogen was identified as severe acute respiratory syndrome coronavirus 2 (SARS-COV-2) (2). According to the Diagnosis and Treatment Protocol for COVID-19 released by the Chinese
National Health Commission (3), based on the severity of presentation, COVID-19 was classified into four categories: (I) mild: clinical symptoms are mild, with no imaging findings suggesting pneumonia; (II) common: fever, respiratory symptoms, and imaging findings are suggesting pneumonia; (III) severe (as long as one satisfied): patients have dyspnea with the respiratory rate (RR) $\geq 30$ times/min, oxygen saturation $\left(\mathrm{SpO}_{2}\right) \leq 93 \%$ at rest, oxygen partial 


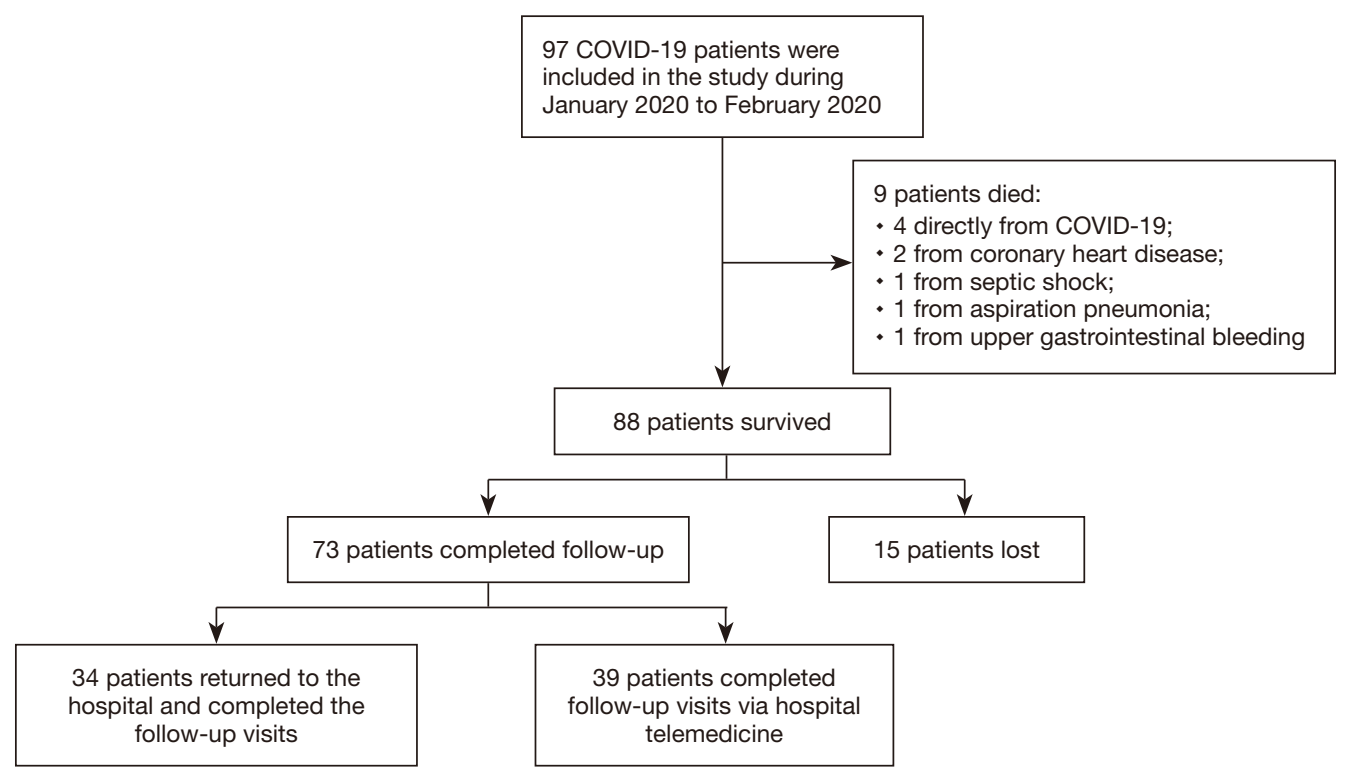

Figure 1 The flow chart of recruiting patients.

pressure $\left(\mathrm{PaO}_{2}\right)$ /oxygen absorption concentration $\left(\mathrm{FiO}_{2}\right)$ $\leq 300 \mathrm{mmHg}(1 \mathrm{mmHg}=0.133 \mathrm{kPa})$; (IV) critical: patients develop respiratory failure requiring mechanical ventilation, shock, or any other organ failure.

Although the biological characteristics of SARSCOV-2 and the clinical features of COVID-19 had been reported extensively (4), there have been limited reports on survival and follow-up of COVID-19 patients, the longterm prognosis of COVID-19 patients is still unclear. In the present study, we aim to compare the clinical characteristics between severe and non-severe patient and investigate their survival information during the followup. We present the following article in accordance with the STROBE reporting checklist (available at https://dx.doi. org/10.21037/apm-21-393).

\section{Methods}

The retrospective study was compliance with the Helsinki Declaration (as revised in 2013) and approved by the Ethics Committee of Beijing Youan Hospital, Capital Medical University (No. 2020-031), and informed consent was waived. From January to February 2020, a number of 97 COVID-19 patients were admitted to the hospital and were all enrolled in this retrospective study.

A diagnosis of COVID-19 was defined as a positive nucleic acid test. According to the Diagnosis and Treatment
Protocol for COVID-19 released by the Chinese National Health Commission (3), we defined the non-severe COVID-19 group as patients with mild or common presentations; severe COVID-19 group as patients with severe or critical manifestations.

The epidemiological history, past medical history, course of the disease, clinical characteristics, laboratory testing, pulmonary imaging examination, treatment and complications were collected through the electronic medical record system of our hospital. The epidemiological history includes Wuhan residence history, cluster case and closely connected history. The course of disease refers to the date from the onset of clinical symptoms to the date of admission. Clinical symptoms (fever, cough and sputum, fatigue, shortness of breath, dyspnea, anorexia, muscle aches, dry throat, sore throat, nasal congestion and runny nose, dizziness, headache, diarrhea) and laboratory testing by blood drawn [blood routine, C-reactive protein (CRP), liver function, kidney function, myocardial enzyme spectrum, coagulation items, procalcitonin (PCT), arterial blood gas analysis] were also obtained. Complications refer to acute respiratory distress syndrome, shock, myocardial injury, arrhythmia, acute kidney injury and toxic liver injury during the treatment.

The ways we followed up are as follows: if patients could not come to our hospital, staffs in the chronic disease management center would make a telephone call after their discharge on day 7, day 14, and 1, 2, 3, 6, 9, 12 months after (Figure 1). 


\section{Statistical analysis}

Data analysis was performed using SPSS Statistical Software version 22.0 (IBM Corporation, Armonk, New York). All continuous variables were compared using the one-sample Kolmogorov-Smirnov test. The normal distribution continuousness variables were listed as the mean \pm standard deviation, while abnormal distribution variables were expressed as median [interquartile range (IQR)]. Comparisons of the age, disease courses, vital signs, and laboratory results between groups were performed by $t$-test or Mann-Whitney test. The $\chi^{2}$ test or Fisher's exact test was used to compare gender, concomitant diseases, epidemiological information, clinical manifestations, imaging characteristics, and therapeutic strategies. KaplanMeier curves and log-rank test were used to examine the overall survival rate between the two groups. Statistical tests were two-sided, and a $\mathrm{P}$ value $<0.05$ indicated a significant difference.

\section{Results}

\section{General information}

Of 97 patients, 67 were categorized in the non-severe group $(69.1 \%)$, and 30 in the severe group (30.9\%). Among the 97 patients, $32(33.0 \%)$ cases had at least one concomitant disease, such as hypertension, coronary heart disease (CHD), cerebrovascular disease, or chronic obstructive pulmonary disease. Most patients were cluster cases. Compared to the non-severe group, the incidence of dyspnea, anorexia was elevated, and body temperature and respiratory rate were significantly higher $(\mathrm{P}<0.05)$, while $\mathrm{SpO}_{2}$ was significantly lower in the severe group $(\mathrm{P}<0.05$, Table 1$)$.

\section{Laboratory testing}

In the severe group, the incidence of declined lymphocyte count $(83.3 \%$ vs. $44.8 \%, \mathrm{P}<0.01)$, and elevated aspartate aminotransferase (AST) $(53.3 \%$ vs. $19.4 \%, \mathrm{P}<0.01)$ and CRP (96.7\% vs. 67.2\%, $\mathrm{P}<0.01)$ increased. Meanwhile, the clue of increased myocardial enzyme spectrum of creatine kinase- $\mathrm{MB}$ (CK-MB) (4/30), myoglobin (MYO) (16/30), and troponin I (TNI) (7/30) were all in the severe group. Compared with the non-severe group, the absolute value of white blood cells (WBCs), AST, serum creatinine (SCr), CRP, CK-MB, MYO, TNI and PCT were increased, while the lymphocyte count and albumin (ALB) were decreased (Table 1).

\section{Imaging features}

Lung computed tomography (CT) scanning was performed in 95 patients (one pregnancy and one died within 24 hours of admission without CT scan). CT scan showed no signs of pneumonia in 5 mild cases. Among the CT manifestations in the lungs of 90 COVID-19 patients, ground glass density shadow (GGO) is the primary sign, and other signs include mesh shadow, fiber cord shadow, "paving stone" sign, tuberous shadow, or consolidation shadow. The images were generally categorized as (I) simple type of GGO $(11.1 \%$, $10 / 90)$; (II) only consolidation $(1.1 \%, 1 / 90)$, which was a single lesion involving one lung lobe, with air bronchial sign; (III) nodular lesions (2.2\%, 2/90), all showed multiple lesions, with GGO around them, presenting as halo sign; (IV) GGO with meshwork shadow, nodular shadow, fibrous cord shadow and "paving stone" sign (12.2\%, 11/90); (V) GGO with consolidation $(20.0 \%, 18 / 90)$, the GGO was seen around some of the consolidation shadows, showing a halo sign; (VI) multiple imaging signs (53.3\%, 48/90), including GGO, consolidation, mesh shadow, fibrous cord shadow, interlobular septal thickening Figure 2. The comparison of imaging features was listed in Table 2.

\section{Complications and therapeutic strategies}

Complications were included acute respiratory distress syndrome, shock (3 septic shock and 2 cardiogenic shocks), myocardial injury, arrhythmia, acute kidney injury, and toxic liver injury. The incidence of various complications and the proportion of patients receiving glucocorticoid and antibiotic treatment in the severe group was significantly higher than that in the non-severe group $(\mathrm{P}<0.05$, Table 3$)$. Ten patients $(10.3 \%)$ were admitted to the intensive care unit (ICU), seven patients were given endotracheal intubation and mechanical ventilation due to severe hypoxemia and respiratory failure. Four of these seven patients received extracorporeal membrane oxygenation (ECMO) due to cardiopulmonary failure. Also, they received continuous renal replacement therapy (CRRT) due to renal failure or combined treatment (Table 3).

\section{Prognosis and survival analysis}

The average hospitalization period was 14 days. The median nucleic acid test conversion time was 7 days, and the nucleic acid test remained positive at death in 5 patients. A total of 
Table 1 Summary of clinical information of 97 patients

\begin{tabular}{|c|c|c|c|c|}
\hline Variables & Total $(n=97)$ & Non-severe $(n=67)$ & Severe $(n=30)$ & $\mathrm{P}$ \\
\hline Male/female & $43 / 54$ & $27 / 40$ & $16 / 14$ & 0.23 \\
\hline Age (years) & $50.8 \pm 20.2$ & $43.1 \pm 17.3$ & $68.1 \pm 15.0$ & $<0.01$ \\
\hline Hypertension & $24(24.7)$ & 8 (11.9) & $16(53.3)$ & $<0.01$ \\
\hline Diabetes & $8(8.2)$ & $5(7.5)$ & $3(4.5)$ & 0.98 \\
\hline CVD & $4(4.1)$ & $0(0)$ & $4(6.0)$ & 0.01 \\
\hline COPD & $4(4.1)$ & $0(0)$ & $4(6.0)$ & 0.01 \\
\hline CKD & $3(3.1)$ & $1(1.5)$ & $2(3.0)$ & 0.26 \\
\hline Close contact & $49(50.5)$ & $32(47.8)$ & $17(56.7)$ & 0.42 \\
\hline Cluster cases & $68(70.1)$ & $43(64.2)$ & 25 (83.3) & 0.04 \\
\hline \multicolumn{5}{|l|}{ Clinical manifestation } \\
\hline Disease course (days) & $5.0(3.0,7.0)$ & $5.0(3.0,7.0)$ & $5.0(2.0,7.0)$ & 0.90 \\
\hline Diagnostic time (days) & $4.0(3.0,6.0)$ & $5.0(3.0,6.0)$ & $4.0(1.0,7.0)$ & 0.49 \\
\hline Fever & $80(82.5)$ & $52(77.6)$ & $28(93.3)$ & 0.06 \\
\hline Cough & $56(57.7)$ & $36(53.7)$ & $20(66.7)$ & 0.23 \\
\hline Fatigue & $31(32.0)$ & $21(31.3)$ & $10(33.3)$ & 0.85 \\
\hline Rhinorrhea & $8(8.2)$ & $6(9.0)$ & $2(6.7)$ & $>0.99$ \\
\hline Dizziness or headache & $5(5.2)$ & $5(7.5)$ & $0(0)$ & 0.30 \\
\hline Diarrhea & $1(1.0)$ & $1(1.5)$ & $0(0)$ & $>0.99$ \\
\hline $\operatorname{Tmax}\left({ }^{\circ} \mathrm{C}\right)$ & $38.3(37.8,38.8)$ & $38.0(37.4,38.5)$ & $38.6(38.3,39.0)$ & $<0.01$ \\
\hline $\mathrm{RR}(\mathrm{bpm})$ & $20.0(20.0,21.0)$ & $20.0(20.0,21.0)$ & $20.0(20.0,22.0)$ & 0.03 \\
\hline $\mathrm{HR}$ (bpm) & $82.0(79.0,90.0)$ & $82.0(80.0,90.0)$ & $87.0(76.0,100.0)$ & 0.43 \\
\hline MAP (mmHg) & $91.3(84.3,96.0)$ & $88.3(83.3,95.3)$ & $93.3(87.3,100.5)$ & 0.05 \\
\hline $\mathrm{SpO}_{2}(\%)$ & $97.2(94.6,98.1)$ & $97.9(96.3,98.8)$ & $92.8(88.4,96.7)$ & $<0.01$ \\
\hline \multicolumn{5}{|l|}{ Laboratory tests } \\
\hline WBC $\left(10^{9} / L\right)$ & $4.13(3.50,5.72)$ & $4.08(3.46,4.94)$ & $5.79(3.59,7.34)$ & 0.05 \\
\hline Lymphocyte $\left(10^{9} / L\right)$ & $0.99(0.74,1.41)$ & $1.17(0.87,1.61)$ & $0.78(0.53,0.90)$ & $<0.01$ \\
\hline Hemoglobin (g/L) & $135.0(124.5,143.5)$ & $135.0(126.0,144.0)$ & $136.5(122.5,143.5)$ & 0.85 \\
\hline
\end{tabular}

Table 1 (continued) 
Table 1 (continued)

\begin{tabular}{|c|c|c|c|c|}
\hline Variables & Total $(n=97)$ & Non-severe $(n=67)$ & Severe $(n=30)$ & $\mathrm{P}$ \\
\hline ALT (U/L) & $28.0(19.5,46.5)$ & $28.0(19.0,45.0)$ & $30.5(23.0,56.0)$ & 0.13 \\
\hline AST (U/L) & $30.0(22.0,43.5)$ & $27.0(20.0,38.0)$ & $45.5(30.0,68.3)$ & 0.01 \\
\hline TBIL $(\mu \mathrm{mol} / \mathrm{L})$ & $8.9(6.5,12.5)$ & $8.7(6.4,11.4)$ & $11.0(6.9,13.7)$ & 0.08 \\
\hline $\mathrm{SCr}(\mu \mathrm{mol} / \mathrm{L})$ & $64.0(53.5,77.5)$ & $61.0(51.0,74.0)$ & $72.5(56.0,88.5)$ & 0.03 \\
\hline CRP (mg/L) & $16.8(3.4,50.5)$ & $11.1(2.0,21.4)$ & $64.4(25.7,113.6)$ & $<0.01$ \\
\hline PTA (\%) & $75.0(70.5,81.5)$ & $75.0(71.0,82.0)$ & $74.0(68.0,81.3)$ & 0.50 \\
\hline PCT (ng/mL) & $0.11(0.10,0.14)$ & $0.11(0.10,0.13)$ & $0.14(0.11,0.19)$ & 0.01 \\
\hline TNI (ng/mL) & $0.01(0.01,0.02)$ & $0.01(0.01,0.02)$ & $0.02(0.01,0.06)$ & 0.01 \\
\hline
\end{tabular}

All variables were expressed as (IQR) or $\mathrm{n}(\%)$. CHD, coronary heart disease; CVD, chronic cardiovascular disease; COPD, chronic obstructive pulmonary disease; CKD, chronic kidney disease; Tmax, maximum body temperature; RR, respiratory rate; HR, heart rate; MAP, mean arterial pressure; $\mathrm{SpO}_{2}$, oxygen saturation; WBC, white blood cells; PLT, platelets; ALT, alanine aminotransferase; AST, aspartate aminotransferase; TBIL, total bilirubin; ALB, albumin; SCr, serum creatinine; CRP, C-reaction protein; PTA, prothrombin activity; $\mathrm{PCT}$, procalcitonin; CK-MB, creatine kinase-MB; MYO, myoglobin; TNI, troponin I.

9 patients died, including 4 directly from COVID-19, 2 from CHD, 1 from septic shock, 1 from aspiration pneumonia, and 1 from upper gastrointestinal bleeding. The 2- and 4-week survival rates of COVID-19 patients were $97.9 \%$ and $95.9 \%$, respectively. The longest follow-up time was 49 weeks, and the median survival time was 47.0 weeks (95\% CI: 45.1-48.9). Up to the date of the last follow-up, of the 88 surviving patients, 73 patients completed the followup, 34 patients came to the hospital for re-examination, and 39 patients were followed up by telephone as living outside the city. Fifteen patients could not be contacted. KaplanMeier tests showed the median survival time of the severe group was significantly lower than the non-severe group $(\mathrm{P}=0.002)$ (Figure 3).

\section{Discussion}

As a full-blown pandemic, COVID-19 has posed a substantial threat to global public health. In the present study, we found severe patients of COVID-19 were characterized by advanced age, more concomitant diseases and complications, which required advanced life support. During the 1-year follow up, the median survival time was 47 weeks for all patients, and only severe patients died within one month. Therefore, the 1-month and 1-year survival rates of total COVID-19 patients were the same as $95.9 \%$.

It was generally accepted that advanced age was the risk factor of severe COVID-19. And it was reported that the prognosis of older people with comorbidities was low (5). More elderly patients' concomitant with hypertension, CHD or chronic cardiovascular disease (CVD) were in the severe group. It supports the necessity to provide comprehensive treatment plans for elderly patients with basic diseases, such as antihypertensive, hypoglycemic and secondary prevention of coronary heart disease (6). Studies $(7,8)$ also indicated that the morbidity might differ between genders. The higher proportion of male patients was explained as the male had a higher expression of angiotensin-converting enzyme 2 (ACE2) receptors, which led to more susceptible to SARS-Cov-2 (9). However, in this study, no difference was found probably due to the limited samples.

The atypical symptoms lack specificity, which made COVID-19 more insidious and difficult to diagnose. However, gastrointestinal symptoms were more common in the severe group, which was consistent with the previous study (10). The RNA of SARS-COV-2 has been detected in the gastrointestinal tissues of COVID-19 patients; it was 

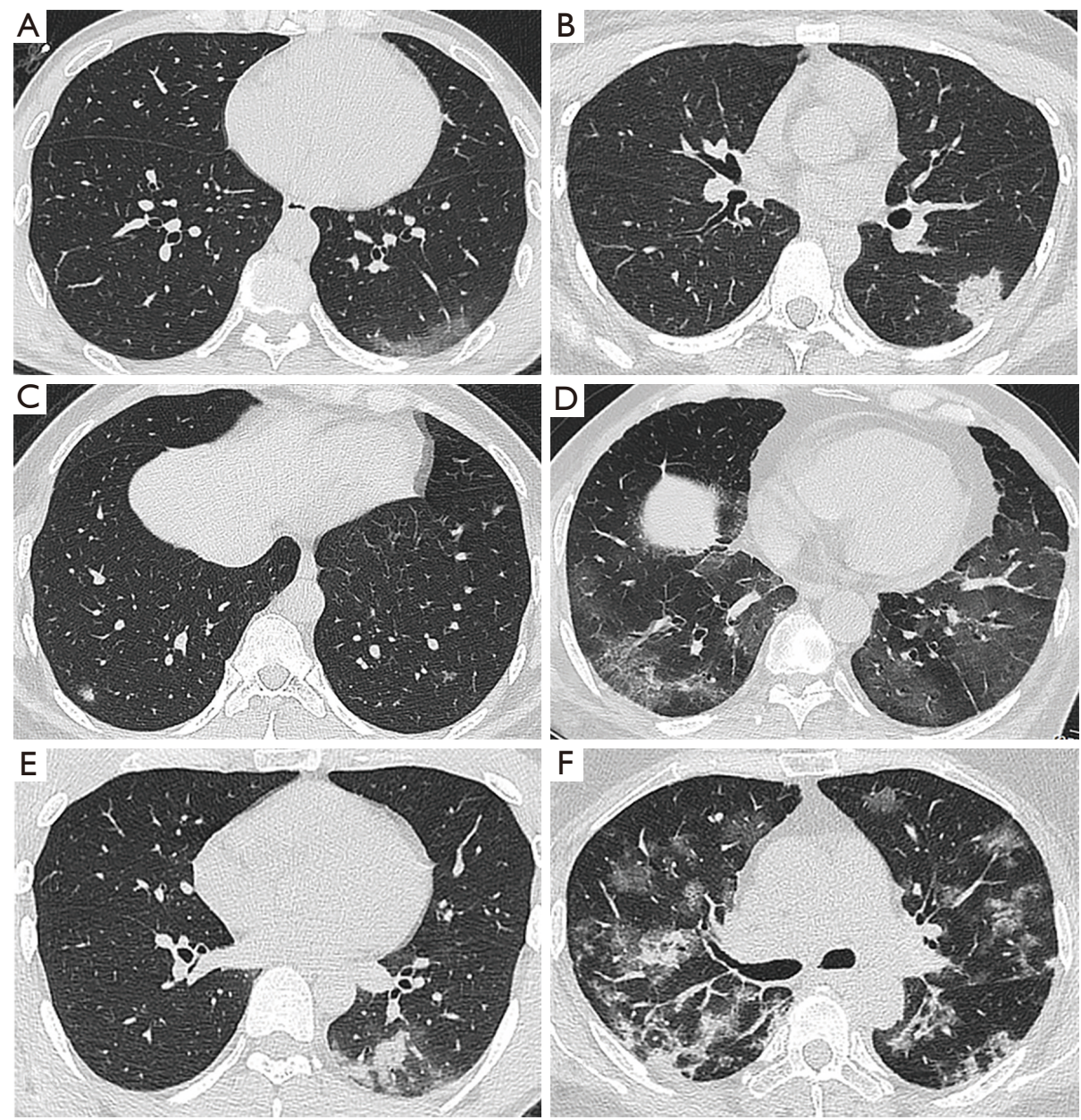

Figure 2 COVID-19 patients with lung CT features. (A) Left lower lobe basal segment with the subpleural pure ground-glass density lesions; (B) complete subpleural consolidation of the posterior outer basement of the lower lobe of the left lung; (C) subpleural nodules were seen in the posterior segment of the lower lobe of the lung, with a "halo" sign around; (D) extensive lesions in both lungs, some with pure GGO or mesh shadow, presenting as "paving stones" sigh; (E) the ground glass density shadow was accompanied by consolidation in the posterior basal segment of the left lower lobe; (F) diffuse lesions in both lungs, manifested as ground glass density shadow with consolidation and broncho vascular bundle thickening.

speculated that the gastrointestinal symptoms of SARSCOV-2 might be related to the invasion of intestinal cells expressing ACE2 (11). Studies have found that patients with gastrointestinal symptoms tend to have a worse prognosis than those without gastrointestinal symptoms (12). Therefore, physicians may pay more attention to gastrointestinal symptoms in the early stage of the disease and find the tendency of severe illness as soon as possible.

For the core imaging features of lung diagnosis, studies have shown that pleural effusion was the typical sign in patients with severe COVID-19 (13). However, this phenomenon was not observed in the present study. In the severe group, pleural thickening with multiple pulmonary lesions involving more than five lobes on both sides and diffuse distribution of simultaneous involvement of lung margins and internal regions were observed.

It was believed that SARS-COV-2 mainly causes tissue damage in patients through direct destruction and immuneinflammatory response. ACE2 was the receptor of SARSCOV-2 (14) and played an essential role in viral infection of host cells. ACE2 has been found to be expressed in lung alveolar type 2 cells, esophageal, gastric, ileum, colon and rectal epithelial cells, renal proximal tubules, hepatobiliary cells, gallbladder epithelial cells, and cardiomyocytes $(15,16)$. 
Table 2 Comparison of imaging characteristics between the two groups

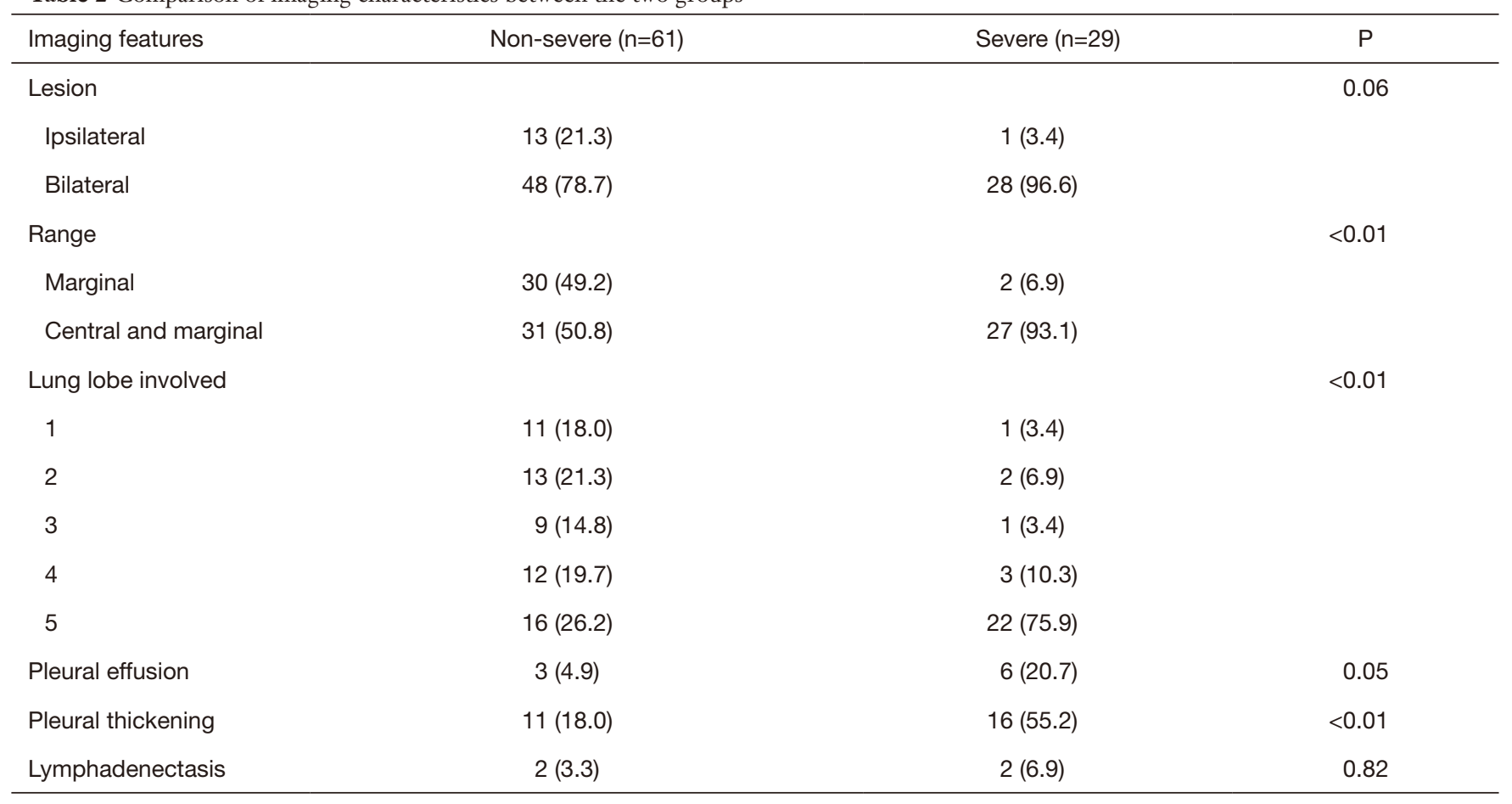

All variables were expressed as $n(\%)$.

Table 3 Treatment and complications between the two groups

\begin{tabular}{|c|c|c|c|c|}
\hline Variables & Total $(n=97)$ & Non-severe $(n=67)$ & Severe $(n=30)$ & $\mathrm{P}$ \\
\hline Glucocorticoid & $26(26.8)$ & $5(7.5)$ & $21(70.0)$ & $<0.01$ \\
\hline Antibiotics & $20(20.6)$ & $6(9.0)$ & $14(46.7)$ & $<0.01$ \\
\hline Noninvasive/high flow oxygen therapy & $3(3.1)$ & $0(0)$ & $3(10.0)$ & 0.03 \\
\hline ECMO & $4(4.1)$ & $0(0)$ & $4(13.3)$ & 0.01 \\
\hline CRRT & $4(4.1)$ & $0(0)$ & $4(13.3)$ & 0.01 \\
\hline ICU Admission & $10(10.3)$ & $0(0)$ & $10(33.3)$ & $<0.01$ \\
\hline \multicolumn{5}{|l|}{ Complications } \\
\hline Myocardial damage & $8(8.2)$ & $1(1.5)$ & 7 (23.3) & 0.01 \\
\hline Arrhythmia & $6(6.2)$ & $1(1.5)$ & $5(16.7)$ & 0.02 \\
\hline Acute kidney injury & $12(12.4)$ & $1(1.5)$ & $11(36.7)$ & $<0.01$ \\
\hline Toxic liver injury & $17(17.5)$ & $7(10.4)$ & 10 (33.3) & 0.01 \\
\hline
\end{tabular}

ECMO, extracorporeal membrane oxygenation; CRRT, continuous renal replacement therapy; ICU, intensive care unit; ARDS, acute respiratory distress syndrome. 


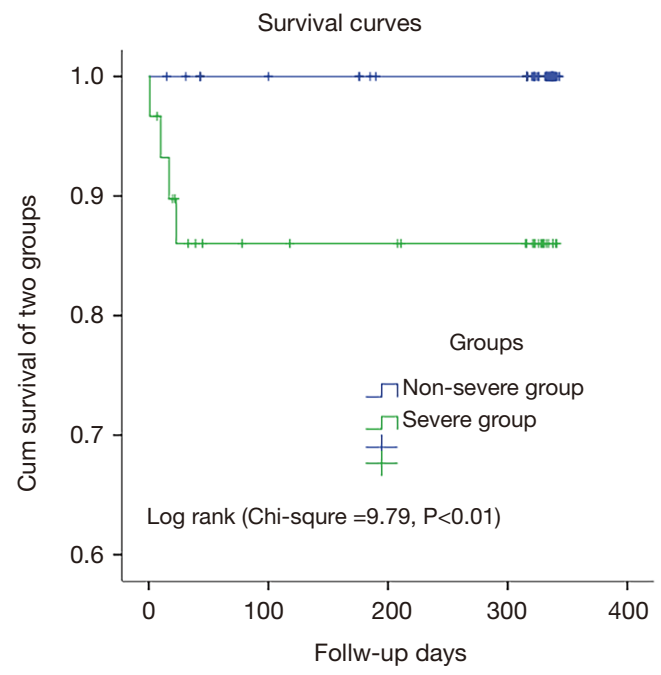

Figure 3 Kaplan-Meier survival curve of COVID-19 patients grouped by disease severity.

Therefore, SARS-COV-2 could attack all these organs and cause tissue damage, leading to corresponding organ dysfunction. It has also been confirmed in our research that patients have abnormal coagulation, myocardial injury, arrhythmia, and even complicated acute respiratory distress syndrome and cardiogenic shock. Pathological anatomy had confirmed that SARS-COV-2 infection could lead to multiple organ damage (17). However, tissue damage was also found in organs that are not directly attacked by the virus. Acute liver damage was one of COVID-19 (4) complications, even though there was no evidence of direct infection of the liver by SARS-COV-2 (17). It had been proposed that patients with severe COVID-19 were more likely to suffer from liver injury (18). However, Bangash et al. (19) stated that severe liver injury was rare in COVID-19 patients. In any case, it was vital to monitor the patients intensively to prevent potential multi-organ injury in severe patients.

Currently, treatment for COVID-19 was still in suspense. The mainstream treatment was still supportive care, including adequate calories, maintaining water and electrolyte homeostasis, and a stable internal environment. While closely monitoring vital signs, detecting early signs of deterioration, treating comorbidities, and providing advanced life support for severe patients. Although the view of glucocorticoids treatment $(20,21)$ was still controversial, $26.8 \%$ of the patients had received glucocorticoids, and most of them were severe patients in the presenting study. Recently, WHO had issued guidelines and recommendations for the treatment of COVID-19 with glucocorticoids, stating that patients with severe and critical COVID-19 could be treated with systemic glucocorticoids for 7-10 days (22).

From this one-year follow-up study, only severe COVID-19 patients died within one month, while after this risk period, no death occurred. It has brought us essential enlightenment that early identification and active treatment for severe patients are crucial for reducing overall mortality. In the analysis of severe acute respiratory syndrome (SARS) and middle east respiratory syndrome (MERS) patients, survivors often suffer from psychological disorders and deterioration in the quality of life $(23,24)$. COVID-19 patients were likely to suffer from long-term quality of life decline due to complications, psychological factors, and loss of social function. Therefore, the assessment of the long-term quality of life of COVID-19 patients warrants further study.

This retrospective study has selection bias because coronavirus patients admitted at designated hospitals and are referred according to administrative requirements, which may affect the patients' outcome. Also, it might have some confounding such as the history of smoking. In addition, all patients in this study were only from one center due to the management of infectious diseases, and we did not follow up on the quality of life of COVID-19 patients.

\section{Conclusions}

In conclusion, severe COVID-19 patients are characterized by advanced age, more concomitant diseases, and complications. According to this 1-year follow-up study, although the survival rate in the severe group was decreased, there were no deaths after one month, which implied a good prognosis if the risk period were passed smoothly.

\section{Acknowledgments}

We thank Dr. Ni Wang and Professor Hui Chen for their statistical analysis. We also thank Dr. Jamie Ward for her help with English writing.

Funding: This work was supported by the Medical Development Fund of China Primary Health Care Foundation-Research and Development and Application of FOLLOW-UP management software for NCP Patients based on Mobile Phone APP (BJYAYY-2020GL-04), and China Primary Health Care Foundation Youan 
Medical Development Fund - Risk Factor Analysis and Early Warning Study of severe COVID-19 (BJYAYY2020ZQN-06).

\section{Footnote}

Reporting Checklist: The authors have completed the STROBE reporting checklist. Available at https://dx.doi. org/10.21037/apm-21-393

Data Sharing Statement: Available at https://dx.doi. org/10.21037/apm-21-393

Conflicts of Interest: All authors have completed the ICMJE uniform disclosure form (available at https://dx.doi. org/10.21037/apm-21-393). The authors have no conflicts of interest to declare.

Ethical Statement: The authors are accountable for all aspects of the work in ensuring that questions related to the accuracy or integrity of any part of the work are appropriately investigated and resolved. The retrospective study was compliance with the Helsinki Declaration (as revised in 2013) and approved by the Ethics Committee of Beijing Youan Hospital, Capital Medical University (No. 2020-031), and informed consent was waived.

Open Access Statement: This is an Open Access article distributed in accordance with the Creative Commons Attribution-NonCommercial-NoDerivs 4.0 International License (CC BY-NC-ND 4.0), which permits the noncommercial replication and distribution of the article with the strict proviso that no changes or edits are made and the original work is properly cited (including links to both the formal publication through the relevant DOI and the license). See: https://creativecommons.org/licenses/by-nc-nd/4.0/.

\section{References}

1. WHO. WHO Coronavirus Disease (COVID-19) Dashboard [EB/OL]. Available online: https://covid19.who.int/

2. Coronaviridae Study Group of the International Committee on Taxonomy of Viruses. The species Severe acute respiratory syndrome-related coronavirus: classifying 2019-nCoV and naming it SARS-CoV-2. Nat Microbiol 2020;5:536-44.

3. National Health Commission. New Coronavirus Pneumonia Diagnosis and Treatment Plan (Trial Version 6) [EB/OL].
Available online: http://www.nhc.gov.cn/xcs/zhengcwj/2020 02/8334a8326dd94d329df351d7da8aefc2.shtml

4. Guan WJ, Ni ZY, Hu Y, et al. Clinical Characteristics of Coronavirus Disease 2019 in China. N Engl J Med 2020;382:1708-20.

5. Chen N, Zhou M, Dong X, et al. Epidemiological and clinical characteristics of 99 cases of 2019 novel coronavirus pneumonia in Wuhan, China: a descriptive study. Lancet 2020;395:507-13.

6. Wang T, Du Z, Zhu F, et al. Comorbidities and multiorgan injuries in the treatment of COVID-19. Lancet 2020;395:e52.

7. Yang X, Yu Y, Xu J, et al. Clinical course and outcomes of critically ill patients with SARS-CoV-2 pneumonia in Wuhan, China: a single-centered, retrospective, observational study. Lancet Respir Med 2020;8:475-81.

8. Nikpouraghdam M, Jalali Farahani A, Alishiri G, et al. Epidemiological characteristics of coronavirus disease 2019 (COVID-19) patients in IRAN: A single center study. J Clin Virol 2020;127:104378.

9. Zhao Y, Zhao Z, Wang Y, et al. Single-cell RNA expression profiling of ACE2, the putative receptor of Wuhan 2019-nCov. bioRxiv 2020. doi: https://doi. org/10.1101/2020.01.26.919985.

10. Wang D, Hu B, Hu C, et al. Clinical Characteristics of 138 Hospitalized Patients With 2019 Novel CoronavirusInfected Pneumonia in Wuhan, China. JAMA 2020;323:1061-9.

11. Fei Xiao, Tang M, Zheng X, et al. Evidence for gastrointestinal infection of SARS-CoV-2. Gastroenterology 2020;158:1831-3.

12. Pan L, Mu M, Yang P, et al. Clinical Characteristics of COVID-19 Patients With Digestive Symptoms in Hubei, China: A Descriptive, Cross-Sectional, Multicenter Study. Am J Gastroenterol 2020;115:766-73.

13. Han R, Huang L, Jiang H, et al. Early Clinical and CT Manifestations of Coronavirus Disease 2019 (COVID-19) Pneumonia. AJR Am J Roentgenol 2020;215:338-43.

14. Walls AC, Park YJ, Tortorici MA, et al. Structure, Function, and Antigenicity of the SARS-CoV-2 Spike Glycoprotein. Cell 2020;181:281-292.e6. Erratum in: Cell. 2020 Dec 10;183(6):1735. doi: 10.1016/j.cell.2020.11.032.

15. Qi F, Qian S, Zhang S, et al. Single cell RNA sequencing of 13 human tissues identify cell types and receptors of human coronaviruses. Biochem Biophys Res Commun 2020;526:135-40.

16. Hikmet F, Méar L, Edvinsson A, et al. The protein expression profile of ACE2 in human tissues. Mol Syst 
Biol 2020;16:e9610.

17. Xu Z, Shi L, Wang Y, et al. Pathological findings of COVID-19 associated with acute respiratory distress syndrome. Lancet Respir Med 2020;8:420-2.

18. Zhang C, Shi L, Wang FS. Liver injury in COVID-19: management and challenges. Lancet Gastroenterol Hepatol 2020;5:428-30.

19. Bangash MN, Patel J, Parekh D. COVID-19 and the liver: little cause for concern. Lancet Gastroenterol Hepatol 2020;5:529-30.

20. Russell CD, Millar JE, Baillie JK. Clinical evidence does not support corticosteroid treatment for 2019-nCoV lung injury. Lancet 2020;395:473-5.

21. Shang L, Zhao J, Hu Y, et al. On the use of corticosteroids for 2019-nCoV pneumonia. Lancet 2020;395:683-4.

Cite this article as: $\mathrm{Li} \mathrm{A}$, Chen F, Gao Y, Huang X, Li T, Zhang J, Liang L, Li X. Clinical features and survival analysis of 97 coronavirus disease 2019 (COVID-19) patients. Ann Palliat Med 2021;10(7):7270-7279. doi: 10.21037/apm-21-393
22. WHO. Corticosteroids for COVID-19. Geneva:World Health Organization 2020. Available online: https:// www.who.int/publications/i/item/WHO-2019-nCoVCorticosteroids-2020.1

23. Ahmed H, Patel K, Greenwood DC, et al. Long-term clinical outcomes in survivors of severe acute respiratory syndrome and Middle East respiratory syndrome coronavirus outbreaks after hospitalisation or ICU admission: A systematic review and meta-analysis. J Rehabil Med 2020;52:jrm00063.

24. Batawi, S, Tarazan, N, Al-Raddadi, R.et al. Quality of life reported by survivors after hospitalization for Middle East respiratory syndrome (MERS). Health Qual Life Outcomes 2019;17:101. 